\title{
Predictive value of red blood cell distribution width in critically ill patients with atrial fibrillation: a retrospective cohort study
}

\author{
Hongfen Zengi", Tingting Tao ${ }^{2 \#}$, Zetao $\mathrm{Ma}^{3,4}$, Min $\mathrm{Wang}^{3,4}$, Xue $\mathrm{Lu}^{3,4}$, Yanbo Zhao ${ }^{3,4 \#}$, Zhida Shen ${ }^{3,4 \#}$ \\ ${ }^{1}$ Department of Operating Room, Sir Run Run Shaw Hospital, Zhejiang University School of Medicine, Hangzhou, China; ${ }^{2}$ Department of \\ Endocrinology, Changxing People's Hospital, Huzhou, China; ${ }^{3}$ Department of Cardiology, Sir Run Run Shaw Hospital, Zhejiang University School \\ of Medicine, Hangzhou, China; ${ }^{4}$ Key Laboratory of Cardiovascular Intervention and Regenerative Medicine of Zhejiang Province, Hangzhou, China \\ Contributions: (I) Conception and design: H Zeng, Y Zhao, Z Shen; (II) Administrative support: None; (III) Provision of study materials or patients: \\ None; (IV) Collection and assembly of data: T Tao, Z Ma; (V) Data analysis and interpretation: Z Shen, H Zeng; (VI) Manuscript writing: All \\ authors; (VII) Final approval of manuscript: All authors. \\ \#These authors contributed equally to this work. \\ Correspondence to: Dr. Yanbo Zhao; Dr. Zhida Shen. Sir Run Run Shaw Hospital, Zhejiang University School of Medicine, Hangzhou 310016 , China. \\ Email: zhaoyb@zju.edu.cn; shenzd@zju.edu.cn.
}

\begin{abstract}
Background Atrial fibrillation (AF) is a leading cause of morbidity and mortality among elderly patients especially for patients in ICU. Previous studies revealed the impact of red blood cell distribution width (RDW) on predicting onset of AF. However, the prognostic value of RDW in critically ill patients with AF remains largely unknown. Thus, this study aims to explore the potential value on predicting in- and out-of-hospital mortality in critically ill patients with AF.
\end{abstract}

Methods: Data were extracted from the Medical Information Mart for Intensive Care (MIMIC) III database and 7,867 critically ill patients with AF were enrolled. The association between RDW and inhospital mortality was evaluated using the multiple logistic regression analysis as a design variable. Shortand long-term outcomes were compared between the low RDW and high RDW groups in critically ill patients balanced by the propensity score matching (PSM) algorithm.

Results: Analysis of the 7,867 patients revealed a linear relationship between RDW and in-hospital mortality. For critically ill patients with AF, the elevated level of RDW was associated with increased inhospital mortality, with the OR increasing from level 2 (OR: 1.75, 95\% CI: 1.25 to 2.44) to level 5 (OR: 3.89, $95 \% \mathrm{CI}: 2.55$ to 5.93$)$ with level $1(\mathrm{RDW} \leq 13)$ as the reference group. 3841 enrolled patients with records in the CareVue systems were selected by PSM algorithm. The baseline characters were well balanced in 1,054 pairs of enrolled patients. A significant lower survival rate was observed in the high RDW group $(\mathrm{P}<0.001)$.

Conclusions: High levels of RDW are associated with increased in- and out-of-hospital mortality in critically ill patients with AF.

Keywords: Red blood cell distribution; atrial fibrillation (AF); propensity score matching (PSM); all-cause mortality

Submitted Aug 25, 2020. Accepted for publication Nov 20, 2020.

doi: 10.21037/apm-20-1704

View this article at: http://dx.doi.org/10.21037/apm-20-1704

\section{Introduction}

Atrial fibrillation (AF) is a leading cause of morbidity and mortality among elderly patients around the world $(1,2)$. In the critically ill patients, AF is the most common arrhythmia (3). Compared with non-AF patients, patients with AF in ICU have worse clinical outcomes (4). Multiple risk factors are found to be highly related to the incidence and clinical outcomes of patients with AF (5). Several studies have estimated the relation between red blood 
cell distribution width (RDW) and AF. RDW is a routine tested hematological test describing the heterogeneity of red blood cell size (6). RDW has been long recognized as an important index for differentially diagnosis of anemia. Recently, several studies revealed the potential role of RDW as a prognostic role in various hematological and nonhematological diseases including AF (7-10). Korantzopoulos et al. reported a higher incidence of new-onset $\mathrm{AF}$ after coronary artery by-pass graft (CABG) surgery in the patients with RDW $>13.3 \%$ (11). Similarly, higher level of RDW was also found in the patients diagnosed of nonvalvular AF (12). Although studies with small sample size have reported the association of increased RDW with worse outcomes in patients, the role of RDW in the critically ill patients with AF remains unclear (13). Therefore, the study between AF and RDW based on sizable samples is beneficial for understanding the outcomes of critically ill patients with $\mathrm{AF}$.

Therefore, in this study, we intended to perform an observational cohort study using the Medical Information Mart for Intensive Care III (MIMIC-III) database to further investigate the internal association between RDW and clinical outcomes of critically ill patients with AF. All the patients diagnosed of $\mathrm{AF}$ were stratified based on first RDW test since ICU admission. The relationship between RDW and in-hospital mortality were discussed. In addition, considering the heterogeneity of patients during ICU admission, a propensity score matching (PSM) analysis was performed. To the best of our knowledge, no previous studies have been conducted on the prognostic value of RDW in critically ill patients with AF.

We present the following article in accordance with the STROBE reporting checklist (available at http://dx.doi. org/10.21037/apm-20-1704).

\section{Methods}

\section{Data source}

This was a retrospective cohort study based on Medical Information Mart for Intensive Care (MIMIC) III (version 1.4) database: a publicly available ICU database published by the Massachusetts Institute of Technology (14). The study was conducted in accordance with the Declaration of Helsinki (as revised in 2013). No informed consent and ethical approval were required because all the data were anonymized. One author (ZS) was approved to access to the database (certification number 8521635) and extracted the data.

\section{Data extraction}

Demographic data, comorbidities, physiological variables during ICU admission, laboratory parameters, sequential organ failure assessment (SOFA) score and the use of indicated agents. The comorbidities of enrolled patients were extracted as previously reported (15). In brief, the ICD 9 codes of selected comorbidities were determined and selected patients were identified using PostgreSQL tools. For deficiency anemia, iron deficiency anemia, folate deficiency anemia, protein deficiency anemia, vitamin B12 deficiency anemia and unspecified deficiency anemia were selected (ICD9 code: 2800, 2801, 2808, 2809, 2811, 2812, 2814, 2818, 2819, 2822, 2823). Vasopressor use was defined as the use of medication use of norepinephrine, phenylephrine, dopamine, isoproterenol, epinephrine, vasopressin or dobutamine while antiarrhythmic drug use was defined as any antiarrhythmic agent use including metoprolol, propranolol, esmolol, atenolol, sotalol, bisoprolol, carvedilol, verapamil, diltiazem, quinidine, flecainide, encainide, propafenone and amiodarone.

\section{Management of missing data}

Missing data were handled according to the missing percentage. For proBNP, more than $20 \%$ were missing and were excluded in the following study. For other continuous variables with missing values than $5 \%$, average or median values were selected to replace the missing values.

\section{Study population and stratification method}

Only the patients of the first ICU study were included in this study. Patients were identified as critically ill patients with AF according to ICD-9 code (ICD-9 code $=4273$ ). Patients who spent less than $24 \mathrm{~h}$ in the ICU were excluded. Besides, patients younger than 18 years or older than 89 years were removed. Finally, the patients with RDW records in ICU were enrolled in this study.

Although previous studies set the normal upper limit of RDW ranging from $11.5 \%$ to $14.5 \%$, the Lowess Smoothing algorithm was used to evaluate the association between hospital mortality and RDW (16). The optimal cutoff value was determined by calculating Youden index of the receiver operating characteristic (ROC) curve. Subsequently, RDW was further categorized into five levels 
for constructing logistic models: level $1(\leq 13)$, level $2(>13$ and $\leq 15)$, level 3 (>15 and $\leq 17)$, level $4(>17$ and $\leq 19)$ and level 5 (>19).

\section{PSM}

PSM was performed to reduce the imbalance between the low RDW and high RDW groups. The baseline characters, comorbidities and indicated drugs were matched using a one-to-one nearest neighbor matching algorithm with a caliper width of 0.05 . After matching, survival analysis was performed to explore the role of RDW in the long-term mortality of critically ill patients with AF. Kaplan-Meier curve was adopted to illustrate the survival status and the significance was compared by log-rank test. Finally, 1,054 matched pairs were generated for further analyses.

\section{Definitions and outcomes}

The primary endpoint was in-hospital mortality, defined as death event before hospital discharge. Secondary endpoints included ICU mortality, hospital and ICU length of stay (LOS). MIMIC contains information collected from Philips CareVue and iMDsoft metaVision systems while CareVue systems contain data of death up to 4 years (17). We also explored the relationship between death after discharge from hospital (28-day mortality, 90-day mortality, 1-year mortality, 4-year mortality) and RDW in the patients from CareVue.

\section{Statistical analysis}

Continuous variables are presented as mean \pm standard deviation (SD) or median (interquartile range). The student's $t$-test, Mann-Whitney U test, Wilcoxon ranksum test was used as appropriate. On the other hand, categorical variables are presented as a percentage and compared using the chi-square test or McNemar test as suitable. Lowess smoother algorithm was used to explore the crude association between RDW and hospital mortality. Multivariate logistic regression models were built by selecting the variables identified from the univariate analyses $(\mathrm{P}<0.20)$. A stepwise backward elimination method was used to remove variables with $\mathrm{P}>0.05$. To avoid hypercollinearity, variables with variance inflation factor (VIF) $\geq 5$ were also removed. The subsequent COX model was constructed based on the variables obtained in the previous logistic model. All statistical analysis was performed using SPSS version 22.0 and STATA 15.0 (SPSS, Chicago, IL, USA; STATA, College Station, TX, USA). All tests were twosided with $\mathrm{P}<0.05$ considered statistically significant.

\section{Results}

\section{Baseline characters}

A total of 7,867 patients were enrolled in the final cohort study while 53,665 patients were excluded including 15,056 repeat ICU admissions, 36,778 patients without AF diagnosis, 938 patients spent less than 24 hours in ICU, 783 patients younger than 18 or older than 89 and 110 patients without recording RDW value during ICU admission. In-hospital death occurred in 1,052 patients while 6,815 patients were still alive at hospital discharge (Figure 1). The initial mortality rate of critically ill patients with $\mathrm{AF}$ was $13.4 \%$. The mean age was $72.3 \pm 11.0$ years and 4,712 patients were male (59.9\%).

The baseline characteristics of the survivors and nonsurvivors are listed in Table 1. First RDW during ICU admission in the table was defined as the initial RDW detection value in routine blood samples while maximum RDW was selected based on all tested RDW values during ICU stay. Compared with survivors, RDW levels were significant higher in the non-survivors group $(\mathrm{P}<0.001)$. SOFA score was significantly higher in non-survivors (6.0, 3.25-9.0) than survivors (4.0, 2.0-6.0). All comorbidities excluding deficiency anemia and diabetes mellitus were significantly between two groups $(\mathrm{P}<0.05)$. Hypertension and coronary artery disease were more common in the survivors while the incidences of other comorbidities were higher in non-survivors. The first $24 \mathrm{~h}$ physiological variables assessed by the monitors in the ICU were also extracted. Generally, non-survivors tended to have higher heart rates, respiratory rates as well as lower blood pressure $(\mathrm{P}<0.05)$. Levels of blood creatine and blood urea nitrogen (BUN) were also significantly higher in the nonsurvivors group. The rate of vasopressor use was higher in the non-survivors than survivors $(60.0 \%$ vs. $48.1 \%$, $\mathrm{P}<0.01)$. Considering anticoagulation therapy was essential for patients diagnosed of AF, we found significant lower usage of warfarin in the non-survivors $(48.1 \% v s .16 .6 \%$, $\mathrm{P}<0.01)$. Similarly, the rate of antiarrhythmic therapy was lower in the non-survivors $(87.5 \%$ vs. $77.9 \%, \mathrm{P}<0.01)$. All these results indicated more critically illness and potential contraindications of routine anticoagulation and antiarrhythmic therapies for patients who had in-hospital 


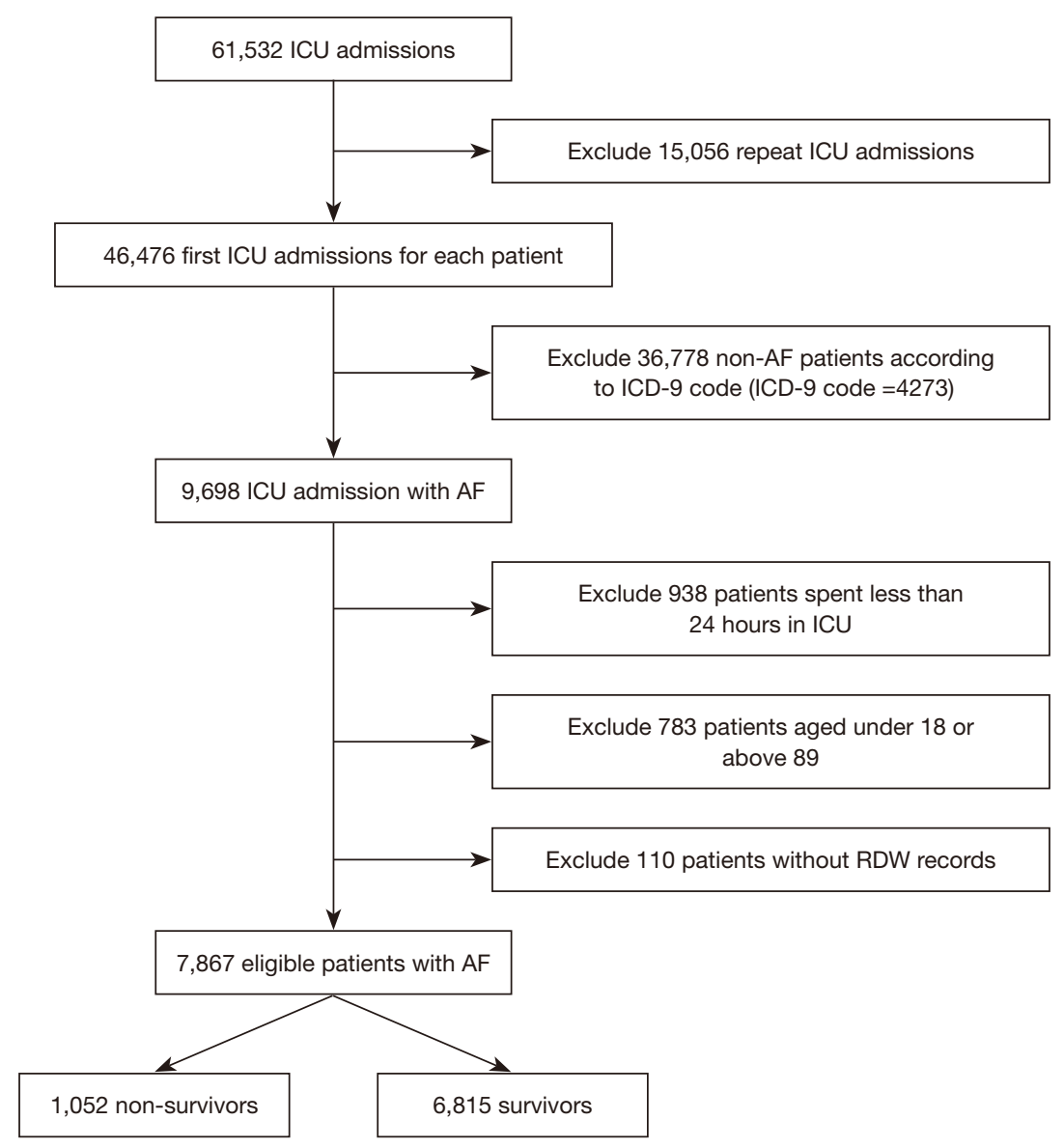

Figure 1 Flow diagram of the study population. AF, atrial fibrillation; ICU, intensive care unit; RDW, red cell distribution width.

death events.

\section{Relationship between RDW and in-hospital mortality}

Next, the relationship between initial RDW and inhospital mortality of patients was explored using the Lowess Smoothing algorithm (Figure 2). For patients diagnosed of AF, in-hospital mortality was positively related with increased RDW value (Figure $2 A$ ). Considering that heart failure and stroke were lethal complications in patients of AF, subgroup analyses were also executed for patients complicated with congestive heart failure or stroke (18). A total of 3,378 patients with congestive heart failure as well as 875 patients with stroke were enrolled for the subgroup analyses. Similarly, elevated RDW levels were correlated with increased in-hospital death events (Figure 2B,C). Moreover, the association between RDW and in-hospital mortality was also investigated in 324 patients with deficiency anemia due to the impact of deficiency anemia on RDW value (Figure 2D) (19). Generally, a linear-shaped relationship between initial RDW and mortality was found for patients with AF. However, the association became less clear in the subgroup analyses partly due to the reduced sample sizes. Therefore, further statistical analyses were executed based on all patients diagnosed of AF.

Due that the linear association was identified, we stratified the patients into two groups by RDW. The best cutoff value was selected using the Youden index by building the ROC curve (Figure S1). Crude outcomes were listed in Table 2. The patients with increased RDW levels had higher hospital mortality $(20.5 \%$ vs. $9.7 \%, \mathrm{P}<0.01)$ as well as ICU mortality $(13.8 \%$ vs. $7.1 \%, \mathrm{P}<0.01)$. Meanwhile, hospital and ICU LOS were also significantly longer in the patients with higher RDW value.

To further explore the impact of RDW, RDW was further stratified into five groups with level $1(\leq 13)$ was selected as the reference. Laboratory parameters were excluding in the multivariate logistic analysis as for the 
Table 1 Baseline characters between in-hospital survivors and non-survivors

\begin{tabular}{|c|c|c|c|c|}
\hline Variables & All patients $(n=7,867)$ & Survivors $(n=6,815)$ & Non-survivors $(n=1,052)$ & $P$ value \\
\hline Age (y) & $72.3 \pm 11.0$ & $71.9 \pm 11.0$ & $74.5 \pm 11.0$ & $<0.001$ \\
\hline Gender (\%, male) & 4,712 (59.9) & $4,131(60.6)$ & 581 (55.2) & 0.001 \\
\hline Weight(kg) & $82.6 \pm 24.1$ & $83.2 \pm 23.8$ & $79.3 \pm 26.1$ & $<0.001$ \\
\hline Elective & $1,757(22.3)$ & $1,695(24.9)$ & $62(5.9)$ & \\
\hline Emergency & $5,879(74.7)$ & 4,927 (72.3) & $952(90.5)$ & \\
\hline Urgent & $231(2.9)$ & $193(2.8)$ & $38(3.6)$ & \\
\hline SOFA score & $4[2-6]$ & $4[2-6]$ & $6(3.25-9)$ & $<0.001$ \\
\hline CAD & $3,353(42.6)$ & $3,079(45.2)$ & $274(26.0)$ & $<0.001$ \\
\hline Heart failure & $3,378(42.9)$ & $2,872(42.1)$ & $506(48.1)$ & $<0.001$ \\
\hline Stroke & $875(11.1)$ & $675(9.9)$ & $200(19.0)$ & $<0.001$ \\
\hline COPD & $1,313(16.7)$ & $1,097(16.1)$ & $216(20.5)$ & $<0.001$ \\
\hline Deficiency anemia & $315(4.0)$ & $278(4.2)$ & $37(3.5)$ & 0.292 \\
\hline CKD & $1,176(14.9)$ & $986(14.5)$ & $190(18.1)$ & 0.002 \\
\hline Diabetes mellitus & $2,346(29.8)$ & $2,054(30.1)$ & $292(27.8)$ & 0.116 \\
\hline Malignancy & $1,215(15.4)$ & $964(14.1)$ & $251(23.9)$ & $<0.001$ \\
\hline $\begin{array}{l}\text { Mean diastolic blood pressure } \\
(\mathrm{mmHg})\end{array}$ & $57.5(52.0-63.8)$ & $57.5(52.2-63.8)$ & $57.1(50.1-63.7)$ & 0.032 \\
\hline \multicolumn{5}{|l|}{ Laboratory parameters } \\
\hline Hemoglobin (g/dL) & $10.5(9.1-12.0)$ & $10.5(9.1-12.0)$ & $10.7(9.3-12.2)$ & $<0.001$ \\
\hline WBC $(K / \mu L)$ & $11.2(8.2-15.3)$ & $11.0(8.1-14.8)$ & $12.9(8.7-17.9)$ & $<0.001$ \\
\hline Plt $(K / \mu L)$ & $184(135-247)$ & $182(134-243)$ & $200(138-283)$ & $<0.001$ \\
\hline $\begin{array}{l}\text { First RDW during ICU admission } \\
(\%)\end{array}$ & $14.5(13.6-15.8)$ & $14.4(13.6-15.6)$ & $15.4(14.1-17.1)$ & $<0.001$ \\
\hline $\begin{array}{l}\text { Maximum RDW during ICU } \\
\text { admission (\%) }\end{array}$ & $15.1(14.1-16.7)$ & $15.0(14.0-15.0)$ & $16.5(14.9-18.5)$ & $<0.001$ \\
\hline proBNP & $4,983[1,900-11,470]$ & $4,742[1,854-10,828]$ & $7,600[2,132-16,441]$ & 0.025 \\
\hline
\end{tabular}

Table 1 (continued) 
Table 1 (continued)

\begin{tabular}{|c|c|c|c|c|}
\hline Variables & All patients $(n=7,867)$ & Survivors $(n=6,815)$ & Non-survivors $(n=1,052)$ & $P$ value \\
\hline Blood creatine (mg/dL) & $1.0(0.7-1.4)$ & $1.0(0.7-1.3)$ & $1.3(0.8-2.0)$ & $<0.001$ \\
\hline Blood urea nitrogen (mg/dL) & $20.0(15.0-30.0)$ & $20.0(14.0-30.0)$ & $30.0(19.0-47.0)$ & $<0.001$ \\
\hline Vasopressor use, n (\%) & $3,911(49.7)$ & $3,280(48.1)$ & $631(60.0)$ & $<0.001$ \\
\hline Antiarrhythmic drug use, n (\%) & $6,786(86.3)$ & $5,966(87.5)$ & $820(77.9)$ & $<0.001$ \\
\hline Time in hospital (days) & $9.0(5.9-14.8)$ & $9.0(6.0-14.5)$ & $8.7(4.5-17.0)$ & 0.01 \\
\hline Time in ICU (days) & $3.1(1.8-6.0)$ & $2.9(1.8-5.3)$ & $5.1(2.6-10.5)$ & $<0.001$ \\
\hline
\end{tabular}

SOFA, sequential organ failure assessment; CAD, coronary artery disease; COPD, chronic obstructive pulmonary disease; CKD, chronic kidney disease; CVP, central venous pressure; WBC, white blood cell; Plt, platelets; RDW, red cell distribution width. proBNP, pro B type natriuretic peptide; ICU, intensive care unit.
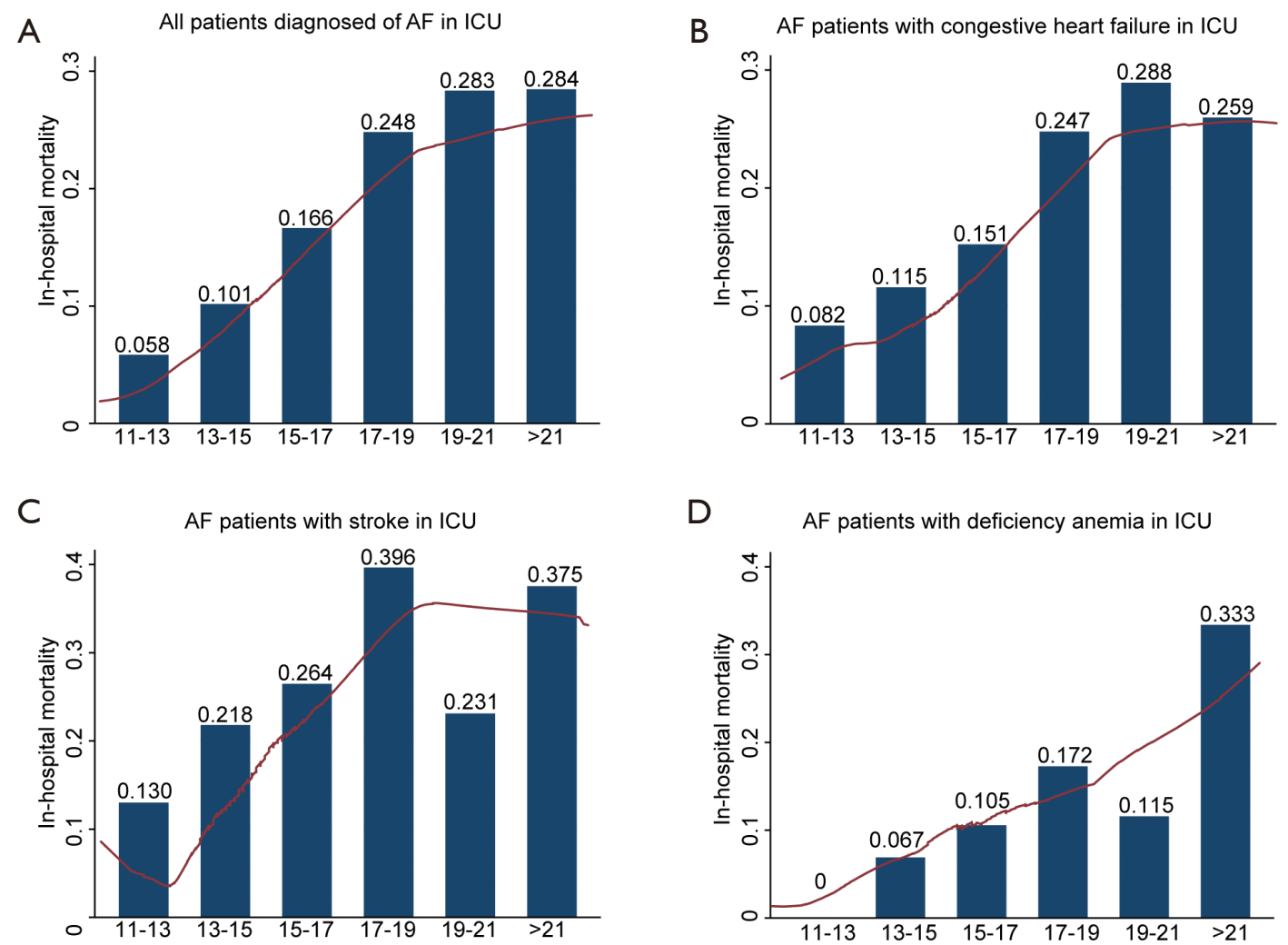

Figure 2 Association between RDW and in-hospital mortality of patients diagnosed of AF (A). Subgroup analyses were executed in patients accompanied with congestive heart failure, stroke, deficiency anemia (B,C,D). In general, linear relationships were identified. AF, atrial fibrillation; ICU, intensive care unit.

missing percentage and hypercollinearity with selected covariates. Figure 3 shows that after adjusting for covariates, elevated RDW level was associated with increased hospital mortality in critically ill patients with AF with the OR increasing steadily from level 2 (OR: $1.75,95 \%$ CI: 1.25 to 2.44) to level 5 (OR: $3.89,95 \%$ CI: 2.55 to 5.93 ). The ORs 
Table 2 Crude outcomes by red blood cell distribution width categories in critically ill patients with AF

\begin{tabular}{|c|c|c|c|c|}
\hline Outcome & All subjects & \multicolumn{3}{|c|}{ Red blood cell distribution width (\%) } \\
\hline Hospital mortality, n (\%) & $1,052(13.4)$ & $506(9.7)$ & $546(20.5)$ & $<0.001$ \\
\hline ICU mortality, n (\%) & $737(9.4)$ & $371(7.1)$ & $366(13.8)$ & $<0.001$ \\
\hline Hospital LOS (days) & $9.0(5.9-14.8)$ & $8.3(5.7-13.4)$ & $10.3(6.3-16.9)$ & $<0.001$ \\
\hline
\end{tabular}

AF, atrial fibrillation; ICU, intensive care unit; LOS, length of stay.

\begin{tabular}{|c|c|c|c|}
\hline \multirow{2}{*}{$\frac{\text { Variables }}{\text { RDW }}$} & Odds Ratio & \multirow[t]{2}{*}{$95 \% \mathrm{Cl}$} & \multirow[t]{2}{*}{$P$ value } \\
\hline & & & \\
\hline RDW (11-13\%) & & $1.00-1.00$ & Ref \\
\hline RDW (13\%-15\%) & $\mapsto \bullet$ & $1.25-2.44$ & 0.001 \\
\hline RDW (15\%-17\%) & $\longmapsto \longrightarrow$ & $1.93-3.86$ & $<0.001$ \\
\hline RDW (17\%-19\%) & & $2.78-5.9$ & $<0.001$ \\
\hline RDW (> 19\%) & 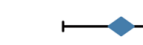 & $2.55-5.93$ & $<0.001$ \\
\hline \multicolumn{4}{|l|}{ Admission type } \\
\hline Elective admission & & $1.00-1.00$ & Ref \\
\hline Emergency admission & & $3.36-5.83$ & $<0.001$ \\
\hline Urgent admission & & $2.07-5.35$ & $<0.001$ \\
\hline SOFA & : & $1.14-1.19$ & $<0.001$ \\
\hline \multicolumn{4}{|l|}{ Comorbidities } \\
\hline COPD & $10-1$ & $1.16-1.66$ & $<0.001$ \\
\hline Stroke & $\mapsto \leftrightarrow$ & $2.46-3.65$ & $<0.001$ \\
\hline Malignancy & $n+-1$ & $1.43-2.05$ & $<0.001$ \\
\hline \multicolumn{4}{|l|}{ Agents use } \\
\hline Antiarrhythmic drugs $\mathrm{H}$ & & $0.43-0.64$ & $<0.001$ \\
\hline Vasopressores & $r-1$ & $1.76-2.45$ & $<0.001$ \\
\hline Warfarin & & $0.19-0.27$ & $<0.001$ \\
\hline
\end{tabular}

Figure 3 Forrest plot of the adjusted ORs from multivariable logistic regression with $95 \%$ confidence interval (CI). The mean variance inflation factor (VIF) was 2.62. RDW, red cell distribution width; SOFA, sequential organ failure assessment; COPD, chronic obstructive pulmonary disease.

of level 4 and level 5 were similar which might be partly related to the small sample size of level 5 .

\section{PSM}

Next, we aimed to discover the role of RDW on shortand long-term outcomes of patients survived during the hospital admission. The CareVue system contains data of death up to 4 years. Therefore, 3,841 patients were enrolled to investigate the short- and long-term outcomes of critically ill patients with $\mathrm{AF}$. The crude survival curves by RDW were presented in Figure 4A. After PSM, 1,054 cases from each group were well matched by a 1:1 matching algorithm (Table 3). The overall propensity score was well balanced after PSM (Figure S2). The heterogeneity between two groups was well minimized with regards to included covariates, such as demographics, comorbidities and indicated agents. After matching, the low RDW groups $(\mathrm{RDW} \leq 15.3)$ had a significantly lower short-term mortality (28-day mortality: $5.1 \%$ vs. $9.0 \%, \mathrm{P}=0.001$; 90 -day mortality: $11.0 \%$ vs. $19.0 \%, \mathrm{P}<0.001$, Table 4). Similarly, 1 -year mortality and 4-year mortality were significantly higher in the patients with high levels of RDW (1-year mortality: $36.1 \%$ vs. $22.1 \%, \mathrm{P}<0.001$; 4 -year mortality: $57.8 \%$ vs. $39.7 \%, \mathrm{P}<0.001)$. The survival rates were significantly lower in the patients with high levels of RDW $(\mathrm{P}<0.001$, Figure 4B).

\section{Discussion}

In the current study, we sought to investigate the relationship between RDW and clinical outcomes of critically ill patients with AF. By using the large free accessible critical care database, we demonstrated that high levels of RDW might be associated with increased in- and out-of-hospital mortality rates in severe cases with AF. First RDW was found to be significantly associated with in-hospital mortality by the multiple logistic regression analysis. Besides, the short- and long-term prediction effects of RDW were also discussed in the surviving patients at discharge. In addition, after adjustment for covariates by PSM, RDW still revealed the good capacity to predict all-cause mortality. To the best of our knowledge, this is the largest cohort study investigating the relation between 

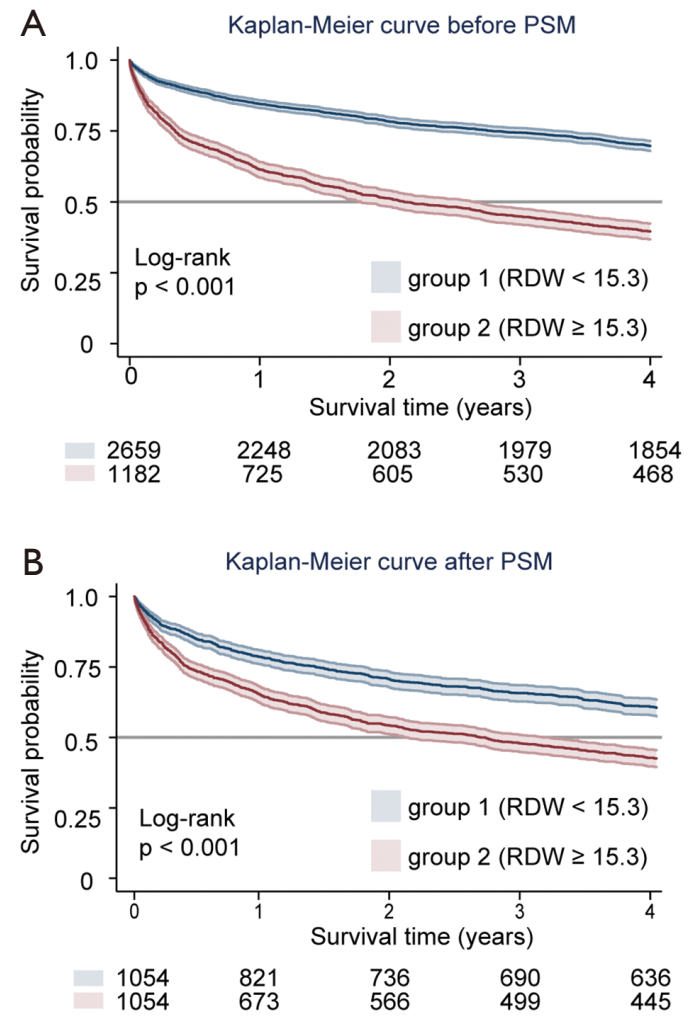

Figure 4 Kaplan-Meier curves before and after propensity score matching (PSM) analysis (A and B). Log rank $\mathrm{P}$ values are indicated in the panels.

RDW and mortality in critically ill patients with AF during mixed ICU admission.

$\mathrm{AF}$ is a usual sustained cardiac arrhythmia especially for the elderly people, with a lifetime morbidity of $25 \%$ (20). The preexistence of $\mathrm{AF}$ is associated with complications of heart failure, stroke and heart failure, which are usually lethal for the patients admitted into the ICU. Among the critically ill patients diagnosed of AF, new-onset AF during ICU admission might indicate the severity of diseases as well as a likely contributor to worse clinical prognosis (4). Besides, anticoagulation management for the patients with new-onset AF during ICU admission is still controversial. The lower rate of warfarin use in the non-survivors might be partly attributed to the different rates of new-onset AF between two groups (Table 1). However, it was difficult to identify the onset time of AF in MIMIC III. Additionally, it was unavailable to determine the presence of $\mathrm{AF}$ in the patients before ICU admission. Therefore, in this study, we investigated the predictive role of RDW in all patients diagnosed of AF during ICU admission regardless of the onset time. Predicting the mortality precisely might be helpful for ICU physicians to optimize the treatment strategy. Therefore, in this study, we explored the potential value of RDW in predicting mortality of critically ill patients with AF. In our study, we found that the value of RDW might be helpful for distinguishing more serious patients. However, the underlying mechanism remained largely unknown. Sustained low-grade inflammation might be an important reason.

There are two requirements for onset of AF: (I) the formation of pathological atrial substrate apt to develop the complex fractionated atrial electrogram (CAFE); and (II) an ectopia arrhythmogenic trigger to induce the outbreak. Acceleration of atrial fibrosis is an important reason of abnormal cardiac substrate remodeling (4).

Overproduced reactive oxygen species (ROS) and inflammation responses are found to be crucial pathological processes for development of atrial fibrosis. Inflammation in patients diagnosed of $\mathrm{AF}$ can arise from different pathways. Patients with $\mathrm{AF}$ are found to be related with low-grade inflammation and increased serum proinflammatory cytokines (21).

On the other hand, RDW levels are found to be related with the degree of inflammation response in multiple diseases. Chronic inflammation was found for patients with malignancy while tumor-related inflammation induced tumorigenesis and progression (22). Previous studies showed RDW was significantly associated with higher IL-6, erythrocyte sedimentation rate (ESR), C-reactive protein (CRP), the inflammatory parameters in patients with malignancy (23). Shi et al. found RDW was related with the incidence rate of colorectal cancer as well as the tumor stages (24). In diffuse large B-cell lymphoma patients, high levels of RDW were an independent poor prognostic factor (25). Similarly, RDW was also associated with nonmalignant diseases. Previous studies showed that RDW could be used to evaluate inflammation response in patients diagnosed of inflammatory diseases, such as obstructive sleep apnea syndrome, sarcopenia, pancreatitis (26-28). In patients with cardiovascular diseases, previous studies also revealed that RDW was a potential diagnostic and prognostic marker. Lippi et al. revealed RDW values had significant correlations with troponin $\mathrm{T}$ levels and had a clinical sensitivity and specificity for diagnosing acute coronary syndrome (29). Meanwhile, elevated RDW levels were related with higher prevalence of acute heart failure (30).

The association between RDW and AF was also discussed in several studies. It was found that the level 
Table 3 Baseline characteristics before and after propensity-score matching in critically ill patients with atrial fibrillation in CareVue systems

\begin{tabular}{|c|c|c|c|c|c|c|}
\hline Variables & \multicolumn{3}{|c|}{ Before PSM } & \multicolumn{3}{|c|}{ After PSM } \\
\hline \multicolumn{7}{|l|}{ Demographics } \\
\hline Age (y) & $71.4 \pm 11.0$ & $72.9 \pm 10.7$ & $<0.001$ & $72.7 \pm 10.8$ & $72.7 \pm 10.8$ & 0.993 \\
\hline Gender (\%, male) & $1,674(63.0)$ & $680(57.5)$ & 0.001 & $595(56.5)$ & $599(56.8)$ & 0.894 \\
\hline Admission type, n (\%) & & & $<0.001$ & & & 0.061 \\
\hline Elective & $752(28.3)$ & $191(16.2)$ & & $179(17.0)$ & $186(17.6)$ & \\
\hline Emergency & $1807(68.0)$ & $948(80.2)$ & & $829(78.7)$ & $827(78.5)$ & \\
\hline Urgent & $100(3.8)$ & $43(3.6)$ & & $46(4.3)$ & $41(3.9)$ & \\
\hline Hypertension & $1,423(53.5)$ & $453(38.3)$ & $<0.001$ & $423(40.1)$ & $431(40.9)$ & 0.742 \\
\hline CAD & $1,322(49.7)$ & $452(38.2)$ & $<0.001$ & 403 (38.2) & $416(39.5)$ & 0.577 \\
\hline Heart failure & $1,033(38.8)$ & $686(58.0)$ & $<0.001$ & $585(55.5)$ & $589(55.9)$ & 0.817 \\
\hline Stroke & $256(9.6)$ & $68(5.8)$ & $<0.001$ & $58(5.5)$ & $63(6.0)$ & 0.702 \\
\hline COPD & $354(13.3)$ & 251 (21.2) & $<0.001$ & 207 (19.6) & 210 (19.9) & 0.821 \\
\hline Deficiency anemia & $62(2.3)$ & $85(7.2)$ & $<0.001$ & $52(4.9)$ & $61(5.8)$ & 0.431 \\
\hline CKD & $151(5.7)$ & $216(18.3)$ & $<0.001$ & $132(12.5)$ & 139 (13.2) & 0.668 \\
\hline Diabetes mellitus & $667(25.1)$ & $425(36.0)$ & $<0.001$ & $345(32.7)$ & $354(33.6)$ & 0.703 \\
\hline
\end{tabular}

PSM, propensity score matching; SOFA, sequential organ failure assessment; CAD, coronary artery disease; COPD, chronic obstructive pulmonary disease; CKD, chronic kidney disease; RDW, red cell distribution width.

of RDW was elevated significantly in patients with paroxysmal non-valvular AF (12). The value of RDW for predicting clinical outcomes was also discussed in several studies $(13,31)$. However, the small sample size of previous studies restricted the findings. In addition, the patients who admitted into ICU had different surroundings and encountered more complex problems. Hence, we studied the role of RDW in critically patients with AF. By using the large public critical care dataset, a big advantage in our study is the large sample size, which allowed for subgroup analysis and subsequent PSM for confounding covariates. The Lowess Smoothing algorithm showed the linear correlation between RDW and in-hospital mortality. The finding was further proved by logistic regression models. In addition, we also evaluated the prognostic value of RDW in patients alive at discharge. Increased RDW was associated with all-cause mortality in the subpopulation. Above all, we concluded that RDW might be a potential marker for evaluating the outcomes of critically ill patients with AF.

Although the large sample size was adopted to adjust for confounding factors, the study had several limitations. First, our study was a single-center retrospective study and the findings should be further confirmed by multicenter prospective studies. Second, due to the retrospective nature, some related clinical variables were unavailable or missing too much. For example, the pathophysiology 
Table 4 Outcomes of patients in matched cohort

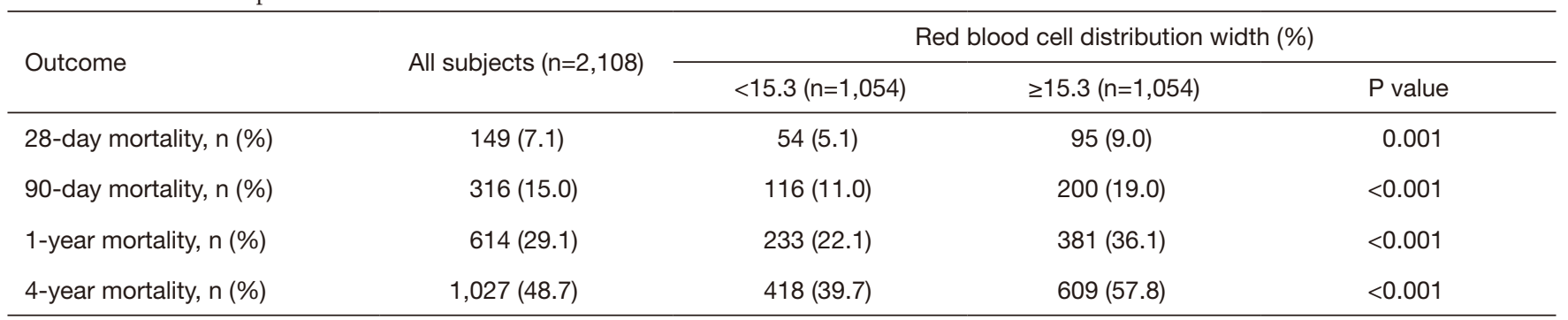

and management of new-onset AF during ICU admission might be different from the sustained AF before ICU stay. Besides, due that our data were retrieved from the public database MIMIC III, the reasons for ICU admission were unavailable, which might be important confounders in the current study. Third, although a lot of covariates were well balanced by PSM, confounders which were not included in our study still existed. Well-designed randomized controlled studies might be the only solution. Besides, in our study, we used the first RDW value instead of maximum RDW. Although maximum RDW value might have a stronger predictive value of all-cause mortality, it was still debating to measure RDW multiple times for predicting the death events more accurately. Lastly, we discussed the association between RDW and all-cause mortality. Some related clinical outcomes were not taken into consideration, such as stroke and readmission events.

\section{Conclusions}

Our study illustrated that RDW was a potential prognostic marker for critically ill patients with AF. Increased RDW was associated with in-hospital mortality as well as the short- and long-term mortality in these patients. Further prospective studies with more clinical details were needed for better predicting the prognostic value. Meanwhile, it was also interesting to discuss the association between RDW and other clinical outcomes such as stroke and readmission to ICU.

\section{Acknowledgments}

We would like to thank Dr. Yan-Fei Shen for statistical assistance.

Funding: This work was supported by the National Natural Science Foundation of China (No. 81800212 and No. $82000404)$ and a grant from the Natural Science Funds of Zhejiang Province, China (Project No. LY17H020002 and Project No. LQ21H020003). The funders had no role in study design, data collection and analysis, manuscript preparation, or decision to publish.

\section{Footnote}

Reporting Checklist: The authors have completed the STROBE reporting checklist. Available at http://dx.doi. org/10.21037/apm-20-1704

Peer Review File: Available at http://dx.doi.org/10.21037/ apm-20-1704

Conflicts of Interest: All authors have completed the ICMJE uniform disclosure form (available at http://dx.doi. org/10.21037/apm-20-1704). All authors report grants from National Natural Science Foundation of China, grants from Natural Science Funds of Zhejiang Province, during the conduct of the study.

Ethical Statement: The authors are accountable for all aspects of the work in ensuring that questions related to the accuracy or integrity of any part of the work are appropriately investigated and resolved. The study was conducted in accordance with the Declaration of Helsinki (as revised in 2013). No informed consent and ethical approval were required because all the data were anonymized. The datasets used and/or analyzed in the present study are available from the corresponding author on reasonable request.

Open Access Statement: This is an Open Access article distributed in accordance with the Creative Commons Attribution-NonCommercial-NoDerivs 4.0 International License (CC BY-NC-ND 4.0), which permits the noncommercial replication and distribution of the article with 
the strict proviso that no changes or edits are made and the original work is properly cited (including links to both the formal publication through the relevant DOI and the license). See: https://creativecommons.org/licenses/by-nc-nd/4.0/.

\section{References}

1. Li H, Gu Y, Liu M, et al. The relationship between red blood cell distribution width and atrial fibrillation in Asian population: A cross-sectional study. Pacing Clin Electrophysiol 2019;42:1197-203.

2. Benjamin EJ, Wolf PA, D'Agostino RB, et al. Impact of atrial fibrillation on the risk of death: the Framingham Heart Study. Circulation 1998;98:946-52.

3. Moss TJ, Calland JF, Enfield KB, et al. New-Onset Atrial Fibrillation in the Critically Ill. Crit Care Med 2017;45:790-7.

4. Bosch NA, Cimini J, Walkey AJ. Atrial Fibrillation in the ICU. Chest 2018;154:1424-34.

5. Parizadeh SM, Jafarzadeh-Esfehani R, Bahreyni A, et al. The diagnostic and prognostic value of red cell distribution width in cardiovascular disease; current status and prospective. Biofactors 2019;45:507-16.

6. Lippi G, Plebani M. Red blood cell distribution width (RDW) and human pathology. One size fits all. Clin Chem Lab Med 2014;52:1247-9.

7. Huang YL, Han ZJ, Hu ZD. Red blood cell distribution width and neutrophil to lymphocyte ratio are associated with outcomes of adult subarachnoid haemorrhage patients admitted to intensive care unit. Ann Clin Biochem 2017;54:696-701.

8. Han YQ, Zhang L, Yan L, et al. Red blood cell distribution width predicts long-term outcomes in sepsis patients admitted to the intensive care unit. Clin Chim Acta 2018;487:112-6.

9. Wang B, Aihemaiti G, Cheng B, et al. Red Blood Cell Distribution Width Is Associated with All-Cause Mortality in Critically Ill Patients with Cardiogenic Shock. Med Sci Monit 2019;25:7005-15.

10. Jia L, Cui S, Yang J, et al. Red blood cell distribution width predicts long-term mortality in critically ill patients with acute kidney injury: a retrospective database study. Sci Rep 2020;10:4563.

11. Korantzopoulos P, Liu T. RDW as a marker of postoperative atrial fibrillation. Int J Cardiol 2015;191:109.

12. Liu T, Shao Q, Miao S, et al. Red cell distribution width as a novel, inexpensive marker for paroxysmal atrial fibrillation. Int J Cardiol 2014;171:e52-3.
13. Wan H, Yang Y, Zhu J, et al. The relationship between elevated red cell distribution width and long-term outcomes among patients with atrial fibrillation. Clin Biochem 2015;48:762-7.

14. Johnson AE, Pollard TJ, Shen L, et al. MIMIC-III, a freely accessible critical care database. Sci Data 2016;3:160035.

15. Feng M, McSparron JI, Kien DT, et al. Transthoracic echocardiography and mortality in sepsis: analysis of the MIMIC-III database. Intensive Care Med 2018;44:884-92.

16. Yu XS, Chen ZQ, Hu YF, et al. Red blood cell distribution width is associated with mortality risk in patients with acute respiratory distress syndrome based on the Berlin definition: A propensity score matched cohort study. Heart Lung 2020;49:641-5.

17. Huang YL, Badrick T, Hu ZD. Using freely accessible databases for laboratory medicine research: experience with MIMIC database. J Lab Precis Med 2017;2:31.

18. Zimetbaum P. Atrial Fibrillation. Ann Intern Med 2017;166:ITC33-ITC48.

19. Salvagno GL, Sanchis-Gomar F, Picanza A, et al. Red blood cell distribution width: A simple parameter with multiple clinical applications. Crit Rev Clin Lab Sci 2015;52:86-105.

20. Lloyd-Jones DM, Wang TJ, Leip EP, et al. Lifetime risk for development of atrial fibrillation: the Framingham Heart Study. Circulation 2004;110:1042-6.

21. Hu YF, Chen YJ, Lin YJ, et al. Inflammation and the pathogenesis of atrial fibrillation. Nat Rev Cardiol 2015;12:230-43.

22. Hanahan D, Weinberg RA. Hallmarks of cancer: the next generation. Cell 2011;144:646-74.

23. Mantovani A, Allavena P, Sica A, et al. Cancer-related inflammation. Nature 2008;454:436-44.

24. Shi C, Xie M, Li L, et al. The association and diagnostic value of red blood cell distribution width in colorectal cancer. Medicine (Baltimore) 2019;98:e15560.

25. Li M, Xia H, Zheng H, et al. Red blood cell distribution width and platelet counts are independent prognostic factors and improve the predictive ability of IPI score in diffuse large B-cell lymphoma patients. BMC Cancer 2019;19:1084.

26. Wu M, Zhou L, Zhu D, et al. Hematological indices as simple, inexpensive and practical severity markers of obstructive sleep apnea syndrome: a meta-analysis. J Thorac Dis 2018;10:6509-21.

27. Kim J, Im JS, Choi CH, et al. The Association between Red Blood Cell Distribution Width and Sarcopenia in U.S. Adults. Sci Rep 2018;8:11484. 
28. Goyal H, Lippi G, Gjymishka A, et al. Prognostic significance of red blood cell distribution width in gastrointestinal disorders. World J Gastroenterol 2017;23:4879-91.

29. Lippi G, Filippozzi L, Montagnana M, et al. Clinical usefulness of measuring red blood cell distribution width on admission in patients with acute coronary syndromes.
Clin Chem Lab Med 2009;47:353-7.

30. Mozos I. Mechanisms linking red blood cell disorders and cardiovascular diseases. Biomed Res Int 2015;2015:682054.

31. Lee KH, Park HW, Cho JG, et al. Red cell distribution width as a novel predictor for clinical outcomes in patients with paroxysmal atrial fibrillation. Europace 2015;17 Suppl 2:ii83-8.

Cite this article as: Zeng $\mathrm{H}$, Tao T, Ma Z, Wang M, Lu X, Zhao Y, Shen Z. Predictive value of red blood cell distribution width in critically ill patients with atrial fibrillation: a retrospective cohort study. Ann Palliat Med 2021;10(3):2469-2480. doi: 10.21037/apm-20-1704 


\section{Supplementary}

A

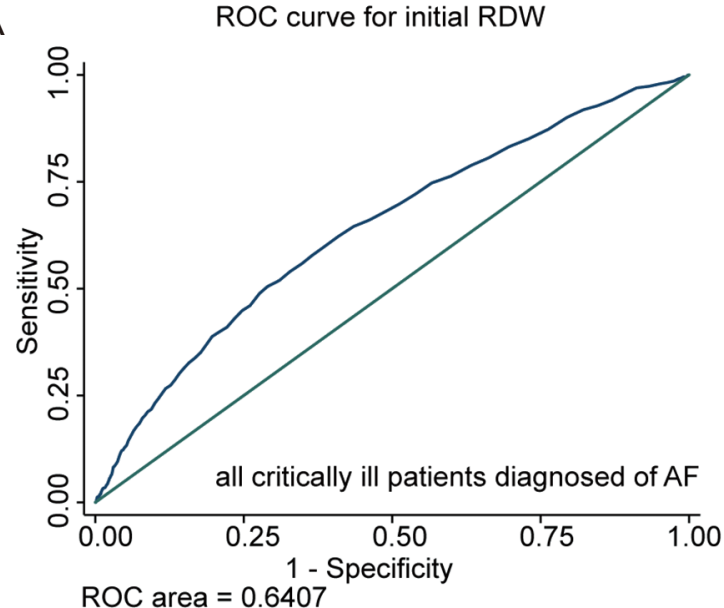

B

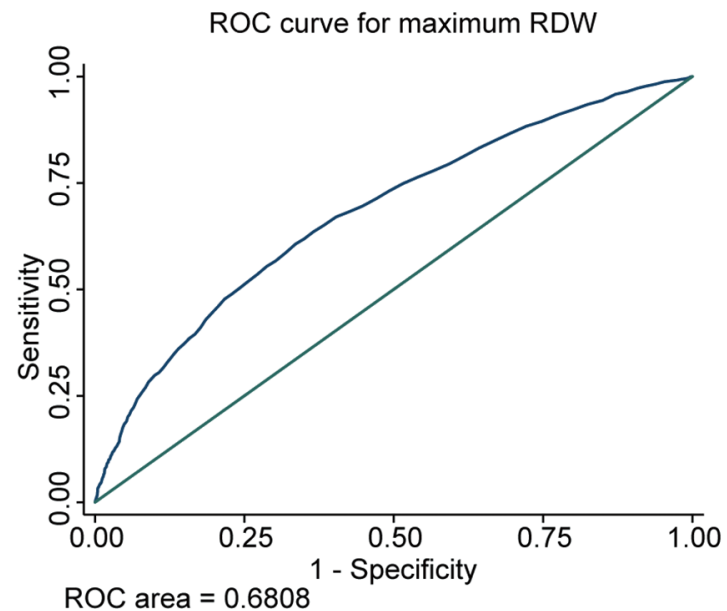

Figure S1 Receiver operating characteristic (ROC) curves for initial and maximum red cell distribution width values during ICU admission.

A

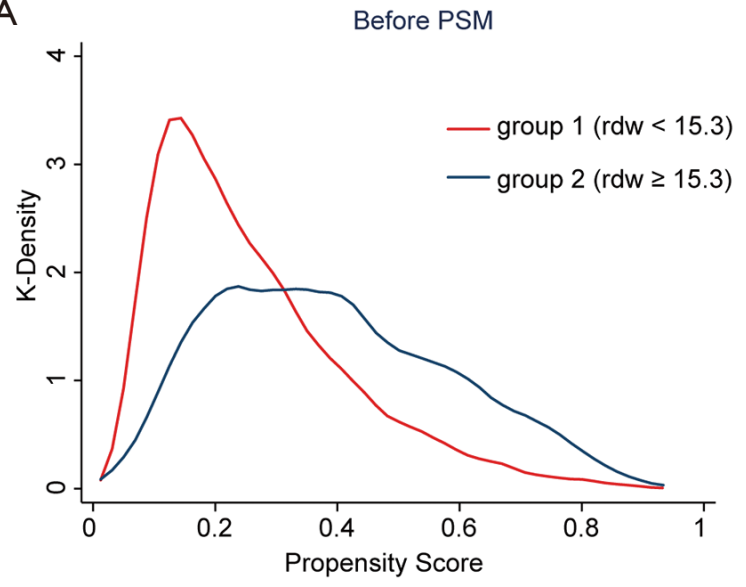

B

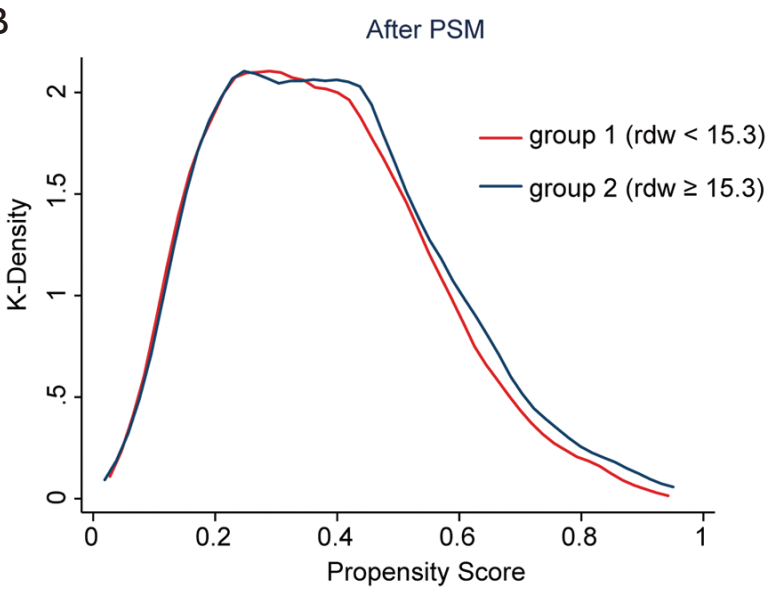

Figure S2 Kernel density plots of the propensity score before and after propensity score matching. 